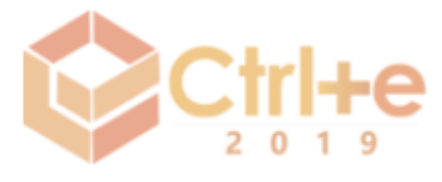

IV Congresso sobre Tecnologias na Educação (Ctrl+E 2019)

Recife, Pernambuco - Brasil

28 a 30 de agosto de 2019

\title{
Tecnologia Digital na Aprendizagem Baseada em Projetos em Curso de Graduação em Enfermagem
}

\author{
Danielle Christine Moura dos Santos1, Raphaela Delmondes do Nascimento1, Jael \\ Maria de Aquino1, Walmir Soares da Silva Júnior1, Marize Conceição Ventin \\ Lima1, Gabrielle Leite Pacheco Lisbôa1, Isabella Almeida1
}

Faculdade de Enfermagem Nossa Senhora das Graças (FENSG) - Universidade de Pernambuco (UPE) - Recife - Pernambuco - Brasil

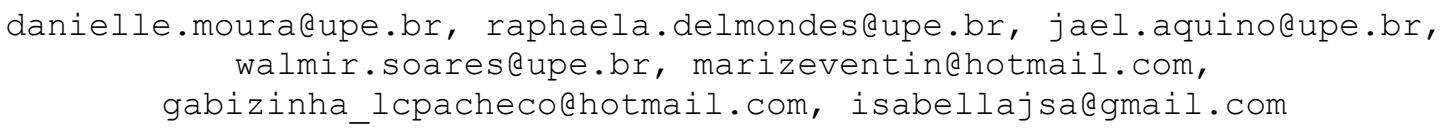

\begin{abstract}
Project-Based Learning (PBL) is a teaching strategy that enables students to confront the issues and problems of a reality they consider significant. The aim of this paper is to report the use of digital technologies in project-based learning in undergraduate nursing. The teaching activities presented compose the contents of scientific methodology of the first period. The results of the activities are presented at a public event organized by students that collaboratively develop the skills expected for the 21st century.
\end{abstract}

Resumo. A Aprendizagem Baseada em Projetos (ABP) é uma estratégia de ensino que consiste em possibilitar que os alunos confrontem as questões e os problemas de uma realidade que consideram significativos. $O$ objetivo deste trabalho é relatar a utilização de tecnologias digitais na aprendizagem baseada em projetos em curso de graduação em enfermagem. As atividades de ensino apresentadas compõem os conteúdos de metodologia científica do primeiro período. Os resultados das atividades são apresentados em evento público organizado pelos estudantes que de modo colaborativo desenvolvem as habilidades esperadas para o século XXI.

\section{Introdução}

Nas últimas décadas, tem-se discutido a necessidade de mudanças no processo de ensino e aprendizagem no ensino superior. No entanto, em sala de aula, o professor, habitualmente, reproduz as mesmas estratégias de ensino às quais foi submetido, em um paradigma tradicional da transmissão [VOSGERAU et al., 2018]

Inovações têm surgido no contexto dos métodos de ensino-aprendizagem, sob influência de pesquisas e modelos comportamentais provenientes da educação, da neurociência e da psicologia. Desataca-se neste cenário, os métodos de aprendizagem ativa, em que o papel do professor passa a ser de um mediador do aprendizado, enquanto o aluno assume o papel de protagonista do processo de aquisição de seu próprio conhecimento [HENRIQUE; PRADO; VIEIRA, 2014]

A aprendizagem ativa (Active Learning) é um termo genérico que expressa as atividades que envolvem os alunos em fazer as coisas e pensar sobre as coisas que eles 
estão fazendo nos processos de aprendizagem. É um método de pedagogia de engajamento, onde busca-se o comprometimento dos alunos na aprendizagem [BONNELL; EISON, 1991]. Ela reúne inúmeras estratégias e métodos de ensino onde se pretende ativar ou desenvolver o pensamento crítico dos alunos no processo de aprendizagem. Como exemplos de metodologias ativas pode-se citar: aprendizagem entre pares, discussões em grupo, estudos de caso (case studies), Flipped Classroom, aprendizagem colaborativa (Cooperative learning), aprendizagem baseada em problemas (PBL - Problem Based Learning), aprendizagem baseada em equipe (TBL - Team Based Learning), aprendizaegm baseada em projetos e inquiry-based learning [GUIMARÃES et al., 2018].

Neste contexto, a Aprendizagem Baseada em Projetos (ABP) é uma estratégia de ensino que consiste em possibilitar que os alunos confrontem as questões e os problemas de uma realidade que consideram significativos, determinando como abordá-los e, então, agindo de forma cooperativa em busca de soluções [BENDER, 2014; TAVARES et al, 2001].

A busca de soluções, de modo cooperativo, pode ser realizado utilizando tecnologias digitais. A inserção da tecnologia nas práticas pedagógicas foi conquistando espaço nos últimos anos, por suas demonstrações e benefícios apresentados. Assim, a integração de aulas presenciais com o uso de tecnologias, vem propiciando $\mathrm{o}$ desenvolvimento da educação em qualquer lugar e a qualquer tempo [ZANOTTO et al, 2014].

A utilização das Tecnologias de Informação e Comunicação (TIC's) é uma interessante metodologia alternativa, unindo o aprendizado e a obtenção de novas informações à boa recepção, por parte dos estudantes com a utilização de ferramentas que aos poucos vem conquistando seu espaço dentro das salas de aula para fins didáticos [LAURILLARD, 2012].

O uso das TIC's na sala de aula exerce um papel cada vez mais importante na comunicação, aprendizado e vivência ao discente. Moran (2015) reforça a necessidade de uma busca constante de transformar o universo educacional em ambientes motivadores e significativos ao aprendizado, acrescentando ao estudante a necessidade de ser: pesquisador, crítico e tomador de decisão pautado nos conhecimentos científicos.

Neste sentido, a Diretriz Curricular Nacional do curso de graduação em enfermagem pauta que é necessário utilizar metodologias ativas de ensino-aprendizagem e abordagens inovadoras que estimulem a aprendizagem significativa, como o uso das diversas tecnologias em favor da educação em saúde. Buscando elaborar projetos educativos de forma participativa com os sujeitos da ação e que contemplem o diagnóstico das necessidades destes, fortalecendo a integração ensino-serviço-comunidade [BRASIL, 2001].

De tal modo, o objetivo deste trabalho é relatar a experiência de um curso de graduação em enfermagem na aplicação de metodologias ativas, destacando a utilização de tecnologias digitais na aprendizagem baseada em projetos.

\section{Método}

Relato de experiência da proposta pedagógica do módulo I (primeiro período), do currículo de enfermagem, da Universidade de Pernambuco, Recife. O currículo passou por uma reforma, e desde 2010 possui estrutura integrada e modular, constituindo-se por 
10 semestres. São desenvolvidas aulas teóricas e práticas, em turno integral, pautado na pedagogia histórico-crítica, com turmas compostas por 60 alunos.

O módulo I tem como objetivo de aprendizagem: compreender a situação de saúde de uma população e a elaborar medidas de organização da atenção básica pela perspectiva do direito à saúde, da justiça social e da equidade, bem como o desenvolvimento de habilidades para o exercício da profissão de enfermagem. Ao final de cada semestre são apresentados Trabalhos de Conclusão de Módulo (TCM) que atendam aos objetivos traçados.

Uma das Unidades temáticas responsáveis pelo TCM é Metodologia Científica, que desde 2014, por meio da Aprendizagem Baseada em Projetos, aborda os conhecimentos básicos sobre elaboração de projetos e relatório de pesquisa buscando integrar todos os conhecimentos desenvolvidos no módulo I nas suas diversas unidades temáticas.

Nas aulas de metodologia científica são utilizadas as ferramentas do G Suite for Education da empresa Google: docs, maps, planilhas e apresentações.

As atividades são fundamentadas na aprendizagem colaborativa conforme apresentado por Dillenbourg (1999), é uma situação de aprendizagem na qual duas ou mais pessoas aprendem ou tentam aprender algo juntas, por meio de atividades presenciais ou virtuais, síncronas ou assíncronas, em um esforço totalmente em conjunto ou com divisão de tarefas. Assim, espera-se que ocorra a aprendizagem como efeito colateral de uma interação entre pares que trabalham em sistema de interdependência na resolução de problemas ou na realização de uma tarefa proposta pelo professor (TORRES; IRALA, 2014)

As turmas com 60 estudantes, geralmente são divididas em grupos de até 10 alunos para as atividades práticas nas comunidades. Esses grupos também são os mesmos para as atividades da unidade temática de metodologia científica e informática em saúde.

\section{Resultados e Discussão}

Ao longo do semestre é realizada a construção do diagnóstico da situação de saúde de uma população residente em um território da cidade do Recife. A cada semestre, é selecionado um novo local, uma comunidade, onde serão realizadas as aulas práticas do módulo. Ao final do módulo, são produzidos relatórios no formato textual e audiovisual com os dados que são coletados nas aulas práticas, e analisados nas aulas de informática em saúde e metodologia científica. Ao final do semestre, serão apresentados em um seminário de integração ensino-serviço.

Essas atividades compõem o planejamento de ensino pautado nas fases da Aprendizagem Baseada em Problemas: 1. Criação e planejamento (onde há a definição de um problema central, a formulação da questão motriz, a seleção das âncoras); 2. Desenvolvimento (envolver a turma no processo, estimular pesquisas, realização do brainstorming); 3. Monitoramento e avaliação (acompanhar e registrar o desenvolvimento dos alunos, verificar os ajustes necessários ao longo do processo); 4. Encerramento (avaliação se os objetivos traçados foram alcançados, divulgação dos resultados/ artefatos). (BENDER, 2014).

O objetivo das atividades das aulas em campo de prática são realizar o levantamento, processamento e descrição da situação de saúde e da organização do trabalho em saúde da família. Todos os dados coletados e analisados são apresentados em 
formato de relatório de pesquisa e pôster que são entregues aos trabalhadores da unidade de saúde da família no Seminário de Integração Ensino e Serviço. Os relatórios colaboram com o planejamento das equipes de saúde, pois apresenta a situação sociodemográfica e de saúde da população local.

Para tal, são realizados nas comunidades selecionadas: 1. Mapeamento do território; 2. Visitas Domiciliares, junto com profissionais de saúde locais, para o preenchimento das fichas de cadastramento familiar e individual. Utiliza-se o prontuário familiar da unidade de saúde, e instrumentos do Ministério da Saúde do sistema de informação da Atenção Básica, o e-SUS; 3. Há entrevistas com as lideranças locais para a compreensão do processo histórico da organização do espaço; 4. Realização de atividade de educação em saúde, planejada após a coleta dos dados e identificação da temática a partir do diagnóstico realizado.

Assim, através de exercícios que fomentem o pensamento científico, crítico e criativo na unidade de metodologia científica e informática em saúde, é lançado o desafio para a turma: Qual a situação de saúde da população alvo? Quais os determinantes e condicionantes do processo de saúde-doença? Essas são as questões motrizes do projeto que será desenvolvido no semestre.

Neste sentido, a trilha de aprendizagem é traçada considerando os conteúdos abordados em metodologia científica: construção de projetos de pesquisa, técnica de coleta, análise e apresentação de dados e informações. Todos os conteúdos são contextualizados a partir do que eles observaram nas visitas realizadas à comunidade. $\mathrm{O}$ objetivo é que esses conteúdos sejam significativos e que estimulem o protagonismo dos alunos na resolução dos problemas.

As atividades de metodologia científica e informática em saúde são pautadas nas habilidades do século XXI, onde os estudantes exercitarão o trabalho em equipe colaborativo, o raciocínio lógico, a comunicação e o manuseio de tecnologias digitais no processo investigativo. É planejado um ambiente de aprendizagem centrado nos alunos, onde utiliza-se pequenos grupos com aprendizagem ativa em que os professores atuem como facilitadores [BENDER, 2014].

Uma das primeiras atividades é a territorialização. A Territorialização é um dos pressupostos básicos do trabalho das equipes de saúde da família. Este processo busca a demarcação de limites de atuação das equipes e o reconhecimento do território e sua dinâmica social. Os estudantes ao percorrerem as ruas da comunidade, registram informações que posteriormente serão analisadas e apresentadas utilizando o Google maps.

Existem vários sites que disponibilizam aplicações envolvendo mapas digitais. Talvez o mais conhecido seja o Google Maps, que a grande maioria das pessoas já utilizou e que serve de base cartográfica para empresas de GPS. O Google Earth é um aplicativo gratuito e fácil de usar, que pode ser de grande utilidade para produção de informação espacial, inclusive nos Programas de Atenção Primária da Saúde.

Para a elaboração dos mapas é utilizado o recurso "My Maps" que possibilita criar mapas personalizados, adicionar pontos ou desenhar formas, criar os mapas com base em planilhas instantaneamente, personalizar o estilo com ícones e cores, adicionar fotos e vídeos a qualquer lugar, e tudo isso pode ser criado de modo colaborativo. Assim, a turma dividida em pequenos grupos, elabora os mapas da comunidade em estudo. Como apresentado na figura 1 , a seguir. 


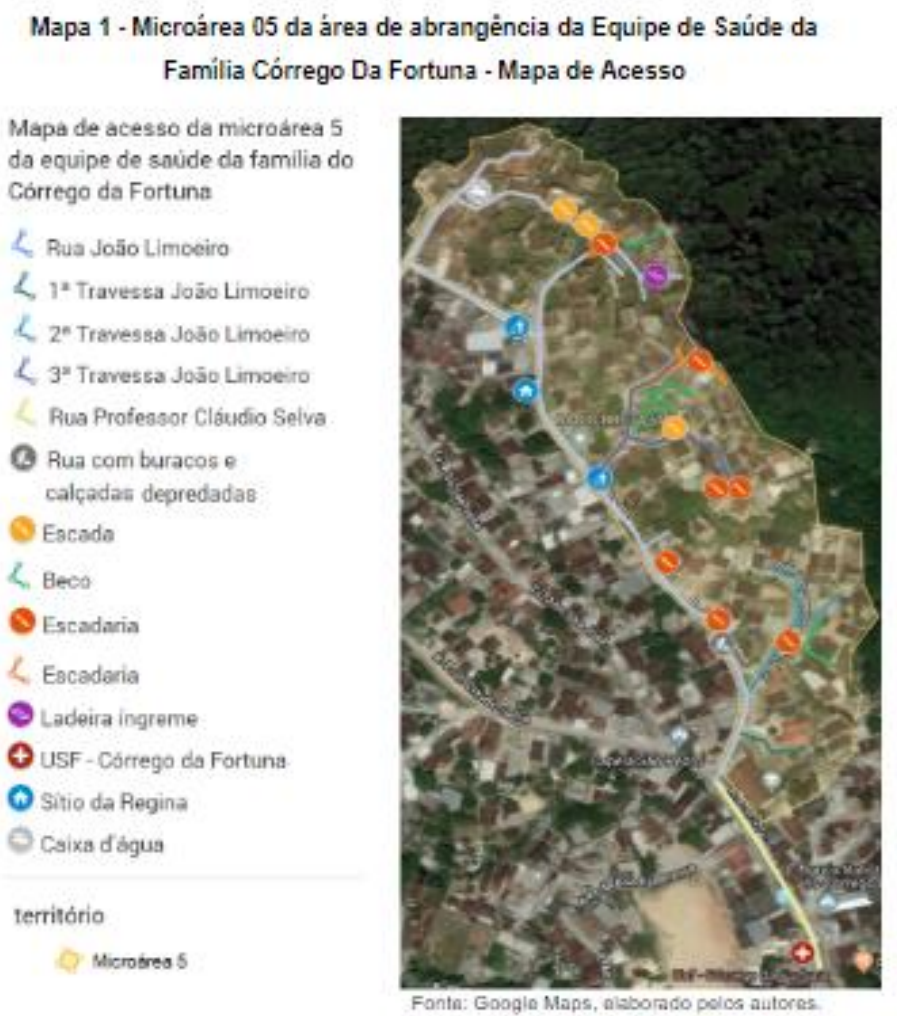

\section{Figura 1 - Mapa de acesso da microárea 05 da abrangência da Equipe de Saúde da Família Córrego da Fortuna, Recife-PE.}

Durante as visitas domiciliares as famílias dos territórios, os estudantes realizam a aplicação de dois formulários que são utilizados no processo de trabalho das equipes de saúde: as fichas e-SUS de cadastro domiciliar e individual.

O e-SUS é uma das estratégias do Ministério da Saúde para desenvolver, reestruturar e garantir a integração dos sistemas, de modo a permitir um registro da situação de saúde individualizado por meio do Cartão Nacional de Saúde. O cadastro domiciliar identifica as características sociossanitárias dos domicílios no território das equipes de $\mathrm{AB}$, este cadastro busca identificar, ainda, situações de populações de rua. Já o cadastro individual identifica as características sociodemográficas, problemas e condições de saúde dos usuários no território das equipes de AB [BRASIL, 2018].

Após a coleta, os dados são consolidados em planilhas, utiliza-se o Google planilhas (sheets) para esta atividade, e que de modo compartilhado e colaborativo cada grupo preenche as informações necessárias no banco de dados. É uma planilha eletrônica de acesso online, que auxilia nas atividades de cálculos e estruturação de dados, a qual permite a acesso e edição dos dados por várias pessoas de modo simultâneo. [MALLMANN; AHLER, 2018].

O Google planilhas permite acessar, criar e editar planilhas no smartphone, tablet ou computador em qualquer lugar, mesmo que não haja uma conexão. E isso facilita o processo de consolidação dos dados, onde todos podem trabalhar juntos $\mathrm{e}$ simultaneamente na mesma planilha, inclusive do acompanhamento dos professores. Outra vantagem desta ferramenta é a sua compatibilidade com o Excel, software da Microsoft [GOOGLE, 2019]. 
Os dados são analisados e são elaborados gráficos e tabelas a partir das variáveis consolidadas. É realizada a produção de relatórios que são apresentados de modo textual segundo as normas da Associação Brasileira de Normas e Técnicas (ABNT), e artísticoculturais, por meio de produções audiovisuais.

Para a elaboração do material textual os estudantes utilizam o Google docs de modo compartilhado nos pequenos grupos e também um relatório final que consolida todos os relatórios de grupos. Para a apresentação dos dados no Seminário, é elaborado material visual utilizando o Google apresentações.

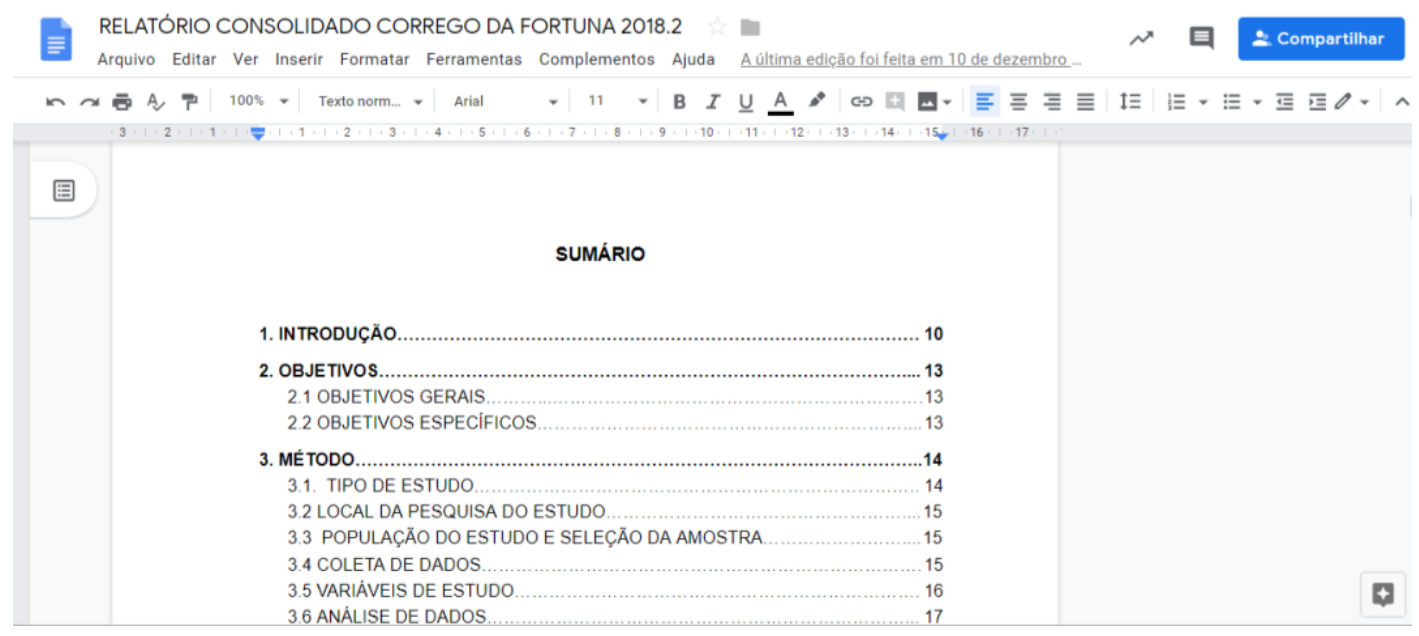

Figura 2 - Relatório consolidado sobre a situação de saúde da população do Córrego da Fortuna, Recife-PE.

Durante a elaboração dos trabalhos, há uma assistência estruturada que é rotineiramente proporcionada pelos professores, com a colaboração de tutores (estudantes de mestrado e doutorado da instituição). Essa atividade consiste nas orientações realizadas em sala de aula, em laboratório de informática e monitoramento dos documentos compartilhados pelos estudantes.

Os documentos são organizados no Google drive compartilhado com todos da turma. O Google drive é destinado ao armazenamento pastas e arquivos na nuvem, permite o compartilhamento destes com demais pessoas, e em diferentes níveis de acesso. Nele são visualizados os documentos em construção [MALLMANN; AHLER, 2018]. Assim, os professores e tutores realizam ajustes, orientações, sugestões de novas fontes de pesquisas, participam dos chats de discussão com os grupos, assim é realizado o feedback e revisões necessárias.

É estimulado que os estudantes criem vídeos com a história da organização do território em análise. Essa atividade tem boa recepção pelos alunos, pois ela envolve criatividade e apresentações persuasivas. São criados vídeos digitais publicados na plataforma YouTube. Para o desenvolvimento de um vídeo coeso, com um bom storyboard, são necessárias habilidades semelhantes as da produção textual.

Ao final do semestre, o trabalho elaborado sobre a descrição de saúde da população da comunidade visitada é apresentado em um Seminário de Integração Ensino e Serviço de Saúde, que conta com a presença de professores de outros módulos, representantes da secretaria de saúde de Recife, trabalhadores da unidade de saúde da comunidade, familiares e amigos dos estudantes. 
Os projetos de $\mathrm{ABP}$ pretendem ser exemplos dos tipos de problemas que os estudantes enfrentam no mundo real, de modo que a apresentação pública dos resultados seja fundamental nesse processo [BENDER, 2014]. Demostrar o produto final, não só os incentiva em melhorar a qualidade do que é elaborado, como também possibilita desenvolver a liderança, a comunicação interpessoal e a oratória, competências essenciais para o mundo do trabalho.

Os estudantes formam uma comissão organizadora do evento, que possui cerimonial para a organização do coffee-break, apresentação do relatório final, apresentações artístico-culturais (dança, música, cordel, vídeos...), homenagens a membros da comunidade, da unidade de saúde, a professores. Eles são apenas orientados durante o processo, mas as ideias para o planejamento e execução partem da turma. $\mathrm{O}$ objetivo é desenvolver esse protagonismo do estudante nas atividades acadêmicas.

O evento reúne em média 150 pessoas a cada semestre, e é o encerramento da unidade temática de metodologia científica e de todo o módulo I. Os relatórios produzidos são entregues aos representantes da Secretaria de Saúde e Unidade de Saúde da comunidade, assim como as demais produções como banner, cartazes, cordel, entre outros.

\section{Considerações Finais}

O uso das tecnologias digitais na Aprendizagem Baseada em Projetos proporciona maior integração e colaboração entre os estudantes e professores, pois possibilita a aplicação de ferramentas que otimizam atividades como o compartilhamento de documentos, assim como a associação de trabalho síncrono com assíncrono.

As novas tecnologias com as suas funcionalidades de acesso remoto $\mathrm{e}$ colaborativo não presencial possibilitam novas formas de ensino. Um dos desafios é a atualização de professores, por meio de formação continuada nas instituições, para a utilização destas ferramentas, num contexto onde os estudantes que ingressam nas Universidades já são nativos digitais e possuem em seu cotidiano a inserção dessas ferramentas na organização de suas atividades.

O estudo suscita a necessidade estudos futuros acerca do impacto da utilização das ferramentas digitais no processo de formação dos futuros profissionais de saúde em instituições que vêm desenvolvendo metodologias ativas em suas propostas de ensino.

\section{Referências}

Bender, W. N. Aprendizagem baseada em projetos: educação diferenciada para o século XXI. Porto Alegre, Penso, 2014.

Brasil. Ministério da Saúde. Secretaria de Atenção à Saúde. e-SUS Atenção Básica: Manual do Sistema com Prontuário Eletrônico do Cidadão PEC - Versão 3.1 [recurso eletrônico] / Ministério da Saúde, Secretaria de Atenção à Saúde, SecretariaExecutiva. - Brasília: Ministério da Saúde, 2018

Bonnell, C. C.; Eison, J. A. Active learning: Creating excitement in the classroom. Washington: George Washington University Press, 1991. 
Guimarães DA, Silva ES. Formação em ciências da saúde: diálogos em saúde coletiva e a educação para a cidadania. Ciência saúde colet. 2010;15(5):2551-62.

Google. Planilhas Google. Disponível em <https://www.google.com/intl/pt$\mathrm{BR} /$ sheets/about/>. Acesso em 22 de junho de 2019.

Henriques, V.; Prado, C.; Vieira, A. Editorial convidado: aprendizagem ativa. Rev. Bras. Ensino Fís. vol.36 no.4 São Paulo Oct./Dec. 2014.

Laurillard, D. Teaching as a design science: building pedagogical patterns for learning and technology. New York: Routledge, 2012.

Tavares, O. L. et al. Ambiente de Apoio à Mediação da Aprendizagem: Uma bordagem Orientada por Processos e Projetos. Revista Brasileira de Informática na Educação - Volume 9 - 2001

Torres, Patrícia Lupion; Irala, Esrom Adriano F. Aprendizagem colaborativa: teoria e prática. Complexidade: redes e conexões na produção do conhecimento. Curitiba: Senar, p. 61-93, 2014.

Vosgerau, Dilmeire Sant'Anna R., et al. Caminhos na preparação do professor universitário para incentivar práticas de aprendizagem ativa. Metodologias pedagógicas inovadoras: contextos da educação básica e da educação superior / Eduardo Fofonca (Coord.); Glaucia da Silva Brito, Marcelo Estevam, Nuria Pons Villardel Camas (Orgs.). Curitiba: Editora IFPR, 2018. 183 p. v. 2

Mallmann, R.; AHLER, E.M. Avaliação das ferramentas colaborativas G Suite no meio acadêmico e o papel do docente frente às novas tecnologias de aprendizagem colaborativa. Revista Tecnologias na Educação - Ano 10 - Número/Vol.25 -Julho 2018

Moran, J. Mudando a Educação com Metodologias Ativas. In: Coleção Mídias Contemporâneas. Convergências Midiáticas, Educação e Cidadania: aproximações jovens. Vol. II] Carlos Alberto de Souza e Ofelia Elisa Torres Morales (orgs.). PG: Foca Foto-PROEX/UEPG, 2015.

Zanotto, M. A. C; Bianchi, P. C, F; Silva, A. P. R.; Reali, A. M. M. R. Hibridização do ensino em uma IES: delineamento de ações pedagógicas para adoção de $20 \%$ a distância em cursos de graduação presenciais, das autoras. In. Simpósio Internacional de Educação a Distância. Universidade Federal de São Carlos. 